\title{
A reevaluation of the 2MASS zero points using CALSPEC spectrophotometry complemented with Gaia Data Release 2 parallaxes
}

\author{
J. Maíz Apellániz ${ }^{1}$ and M. Pantaleoni González ${ }^{1,2}$ \\ ${ }^{1}$ Centro de Astrobiología, CSIC-INTA, Campus ESAC Camino bajo del castillo s/n, 28692 Villanueva de la Cañada, Spain \\ e-mail: jmaiz@cab.inta-csic.es \\ 2 Departamento de Astrofísica y Física de la Atmósfera, Universidad Complutense de Madrid, 28040 Madrid, Spain
}

Received 20 July 2018 / Accepted 30 July 2018

\begin{abstract}
Context. 2MASS is the reference survey in the near-infrared (NIR) part of the spectrum due to its whole-sky coverage, large dynamic range, and proven calibration uniformity. However, previous studies disagree on the value of the zero points (ZPs) for its three bands $J H K$ at the hundredth of a magnitude level. The disagreement should become more noticeable now that Gaia provides whole-sky optical photometry calibrated below that level.

Aims. We want to establish the value of the 2MASS ZPs based on NICMOS/HST spectrophotometry of the CALSPEC standard stars and test it with the help of Gaia DR2 parallaxes.

Methods. We have computed the synthetic $J H K$ photometry for a sample of stars using the HST CALSPEC spectroscopic standards and compared it with their 2MASS magnitudes to evaluate the ZPs. We have tested our results by analyzing a sample of FGK dwarfs with excellent 2MASS photometry and accurate Gaia DR2 parallaxes.

Results. The Vega ZPs for 2 MASS $J, H$, and $K$ are found to be $-0.025 \pm 0.005,0.004 \pm 0.005$, and $-0.015 \pm 0.005$ mag, respectively. The analysis of the FGK sample indicates that the new ZPs are more accurate than previous ones.
\end{abstract}

Key words. surveys - methods: data analysis - techniques: photometric

\section{Introduction}

The precise calibration of photometric surveys requires an almost constant reevaluation, as more data are accumulated over time and new surveys introduce new opportunities to test their compatibility. The Two Micron All Sky Survey (2MASS; Skrutskie et al. 2006) revolutionized infrared astronomy in the previous decade, as it introduced what is now the golden standard in near-infrared (NIR) photometry in terms of sky coverage, dynamic range, and stability for its three $J H K$ bands. The recent Gaia Data Release 2 (DR2; Gaia Collaboration 2018) is likely to produce a similar revolution in optical photometry with its whole-sky $G G_{\mathrm{BP}} G_{\mathrm{RP}}$ photometry that will be expanded into spectrophotometric information in future data releases.

In this Letter we calculate new values for the 2MASS $J H K$ Vega zero points (ZPs) using the recently updated CALSPEC library (Bohlin et al. 2017) ${ }^{1}$ in Sect. 2. In Sect. 3 we check these values using FGK dwarfs with Gaia DR2 parallaxes. For consistency with our previous work, we use the Vega spectral energy distribution (SED) provided by Bohlin $(2007)^{2}$ and the 2MASS sensitivity functions provided by the 2 MASS website ${ }^{3}$.

\footnotetext{
1 See also http://www.stsci.edu/hst/observatory/crds/ calspec.html

2 Available from ftp://ftp.stsci.edu/cdbs/calspec/alpha_ lyr_stis_003.fits

3 https://www.ipac.caltech.edu/2mass/releases/allsky/ doc/sec6_4a.html\#rsr
}

The reader can use the appendix if interested in converting the results to other magnitude systems.

\section{New 2MASS Vega zero points from CALSPEC}

The technique we apply in this paper has been used in the past (Maíz Apellániz 2005a, 2006, 2007, 2017) to test different photometric systems. We compute the synthetic photometry of stars observed with high-quality HST spectrophotometry and we compare it with the observed photometry. If the assumed sensitivity function corresponds to the actual one, a plot of the difference between the observed and synthetic magnitudes (or colors) as a function of color should be essentially flat (i.e., have no color terms). The weighted mean of the values in the vertical axis is the $\mathrm{ZP}$ and the standard deviation of their mean its uncertainty. If, on the other hand, a color term is present, one needs to go back to the drawing board and derive a new sensitivity function (Weiler et al. 2018).

We selected from the CALSPEC database those objects with NICMOS spectrophotometry (Bohlin \& Cohen 2008) and 2MASS photometric uncertainties lower than $0.05 \mathrm{mag}$. Those criteria leave 22,21 , and 19 stars for $J, H$, and $K$, respectively (Table 1). The comparison between the observed and synthetic photometry is shown in Fig. 1.

For all three filters we detect no significant color term, as the restricted fit (no color term allowed) is always encompassed by the unrestricted fit in the plotted color range. Therefore, the 
Table 1. CALSPEC sample used for each filter.

\begin{tabular}{lll}
\hline \hline 2MASS ID & CALSPEC file & Filters \\
\hline J05053062+5249519 & g191b2b_stisnic_006.fits & $J H K$ \\
J05522761+1553137 & gd71_stisnic_006.fits & $J H$ \\
J09211915+8143274 & agk_81d266_stisnic_006.fits & $J H K$ \\
J12570233+2201526 & gd153_stisnic_006.fits & $J H$ \\
J13385054+7017077 & grw_70d5824_stisnic_007.fits & $J H K$ \\
J14515797+7143173 & p041c_stisnic_007.fits & $J H K$ \\
J15591357+4736419 & p177d_stisnic_007.fits & $J H K$ \\
J16194609+5534178 & snap2_stisnic_007.fits & $J$ \\
J16313382+3008465 & p330e_stisnic_008.fits & $J H K$ \\
J16553529-0823401 & vb8_stisnic_006.fits & $J H K$ \\
J17325264+7104431 & 1732526_stisnic_004.fits & $J H K$ \\
J17403468+6527148 & 1740346_stisnic_003.fits & $J H K$ \\
J17430448+6655015 & 1743045_stisnic_004.fits & $J H K$ \\
J17551622+6610116 & kf08t3_stisnic_001.fits & $J H K$ \\
J17583798+6646522 & kf06t2_stisnic_004.fits & $J H K$ \\
J18022716+6043356 & 1802271_stisnic_004.fits & $J H K$ \\
J18023073+5837381 & hd165459_stisnic_004.fits & $J H K$ \\
J18052927+6427520 & 1805292_stisnic_004.fits & $J H K$ \\
J18120957+6329423 & 1812095_stisnic_004.fits & $J H K$ \\
J21321623+0015144 & lds749b_stisnic_006.fits & $J$ \\
J22031077+1853036 & hd209458_stisnic_007.fits & $J H K$ \\
J22113136+1805341 & bd_17d4708_stisnic_006.fits & $H K$ \\
J23195840-0509561 & feige110_stisnic_006.fits & $J H K$ \\
\hline
\end{tabular}

published sensitivity functions agree with the data presented here and no new functions need to be calculated. For the restricted fit, we obtain reduced $\chi^{2}$ values of $0.76,0.59$, and 0.75 for $J, H$, and $K$, respectively. Such low values indicate that if anything, the 2MASS uncertainties are slightly overestimated on average for these objects (note that we have not included an uncertainty term from the spectrophotometry, but that would only make the reduced $\chi^{2}$ values even lower).

The values for the ZPs derived from the restricted fit are given in Table 2, along with the equivalent results from previous works. The associated uncertainties for the ZPs obtained here are $0.005 \mathrm{mag}$ in all three cases. The ZP derived for $H$ is consistent with the previous values. The ZPs derived for $J$ and $K$ are both close to the lower end of previous results, with the one for $J$ being closer to our previous results (Maíz Apellániz 2007, which were obtained using a preliminary version of this analysis with a previous version of CALSPEC data) and the one for $K$ being closer to the result of Cohen et al. (2003).

\section{Testing the zero points with FGK dwarfs}

We test our values for the 2MASS ZPs using additional information from Gaia DR2, most importantly the parallaxes $\varpi$. We start by cross-matching the whole 2MASS catalog with Gaia DR2 and selecting only the objects with good-quality photometry in all six bands $G G_{\mathrm{BP}} G_{\mathrm{RP}} J H K$. We then select a sample of lowextinction FGK dwarfs by applying the following restrictions to the sample above:

$-0.0<J-H<0.6$ and $-0.1<H-K<0.2$, which is the region where low-extinction FGK dwarfs are located (Finlator et al. 2000).

- $\varpi / \sigma_{\varpi}>10$, that is, only stars with low relative-uncertainty parallaxes in order to be able to use the approximation for the distance $d=1 / \varpi$ without introducing significant biases (Maíz Apellániz 2001, 2005b; Luri et al. 2018).
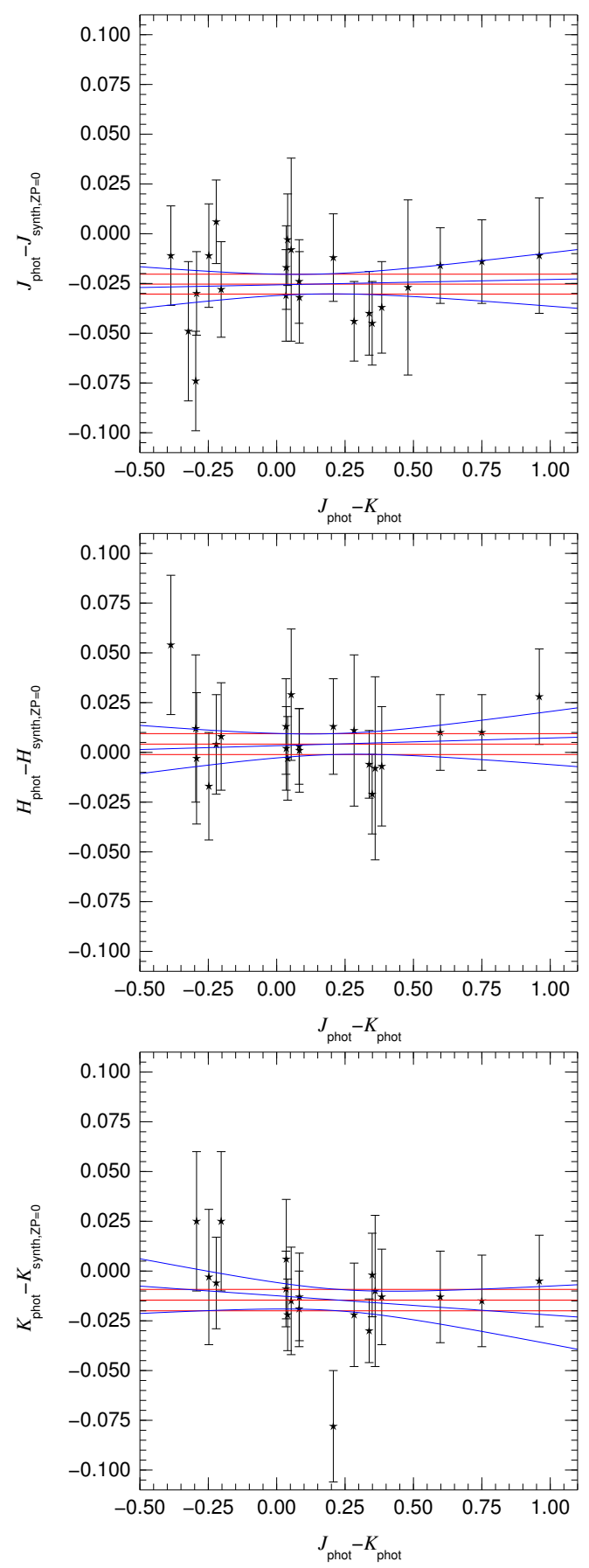

Fig. 1. Difference between the photometric and the synthetic magnitudes as a function of photometric $J-K$ for the CALSPEC stars in this sample assuming a Vega ZP of 0.0 for 2MASS $J$ (top), $H$ (center), and $K$ (bottom). For each plot we show the fit plus uncertainties assuming a linear color term (blue) and no color term (red).

- A double cone in Galactic latitude $|b|>60^{\circ}$ to minimize extinction. We have used Schlafly \& Finkbeiner (2011) and the Maíz Apellániz et al. (2014) extinction law with $R_{5495}=3.1$ to calculate that the average $A_{1}$ (extinction at $1 \mu \mathrm{m})$ at large distances in that region is $0.024 \mathrm{mag}$, which becomes our expected value (within the uncertainties as to the exact location of the dust along the line of sight).

- $\sigma_{J}<0.025 \mathrm{mag}, \sigma_{H}<0.025 \mathrm{mag}, \sigma_{K}<0.025 \mathrm{mag}$ and 2MASS quality flag AAA to minimize noise. 
Table 2. ZPs based on the Bohlin (2007) Vega SED derived in this work and in previous ones.

\begin{tabular}{lccc}
\hline \hline Reference & $J$ & $H$ & $K$ \\
\hline Cohen et al. (2003) & -0.006 & +0.007 & -0.022 \\
Holberg \& Bergeron (2006) & -0.014 & +0.004 & +0.005 \\
Maíz Apellániz (2007) & -0.021 & +0.009 & +0.000 \\
This work & -0.025 & +0.004 & -0.015 \\
\hline
\end{tabular}

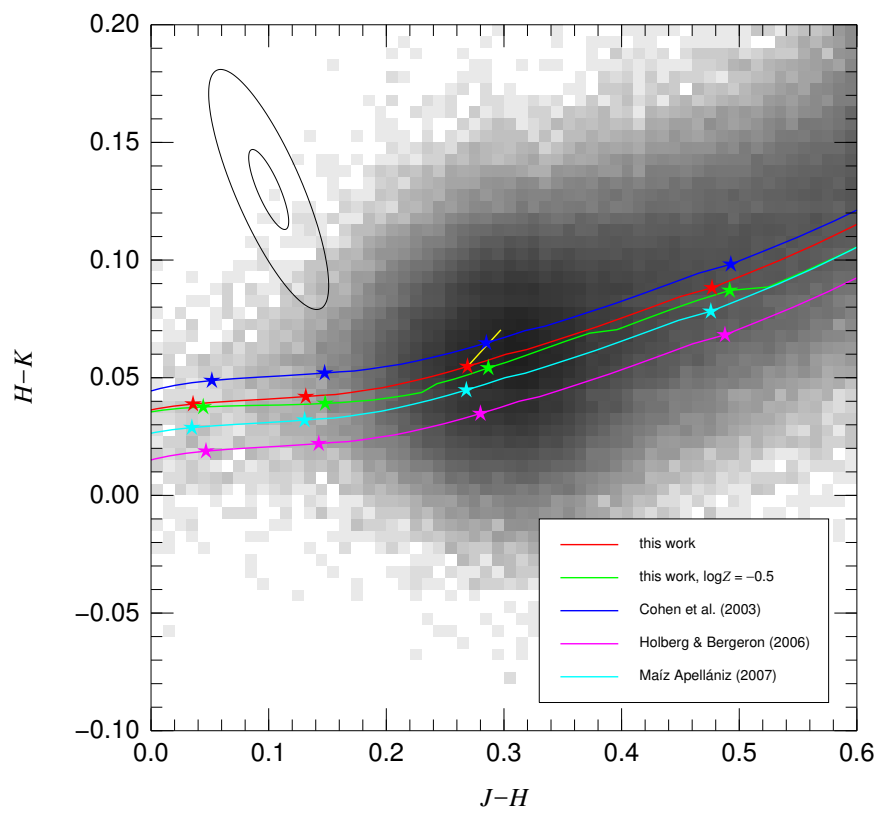

Fig. 2. $J-H+H-K$ density plot for the FGK dwarf sample described in the text. The intensity scale is logarithmic. The colored lines show the location of the Brott \& Hauschildt (2005) dwarf models for four different sets of 2MASS ZPs assuming solar metallicity and for the ZPs in this work assuming $\log Z=-0.5$ in solar units. The colored stars mark the location of the models with $T_{\text {eff }}=8,7,6$, and $5 \mathrm{kK}$ (from left to right), respectively. The large ellipse shows the $68.5 \%$ contour for a distribution assuming $\sigma_{J}=\sigma_{H}=\sigma_{K}=0.0015 \mathrm{mag}$ and the small ellipse the equivalent for the $\mathrm{ZP}$ uncertainties in this work. The straight yellow line shows the displacement that corresponds to an extinction with $A_{1}=0.1 \mathrm{mag}$ and $\alpha=2.0$.

- $100 \mathrm{pc}<d<1000 \mathrm{pc}$ to avoid biases caused by saturation of bright stars and loss of dim stars.

- A cut in $K$ absolute magnitudes $(K-5 \log d+5)$ uncorrected for extinction to exclude subdwarfs and evolved stars.

With those conditions, we are left with a sample of 216393 stars, whose density plot in the $J-H+H-K$ plane is shown in Fig. 2. We also plot there the location of the colors predicted by the Brott \& Hauschildt (2005) SED models with solar metallicity assuming no extinction and the different Vega ZPs in Table 2. To explore the (small) effect of metallicity on intrinsic colors we show the case of $\log Z=-0.5$ (solar units) for the ZPs in this work.

Most of the dispersion in Fig. 2 is caused by the photometric uncertainties (see large ellipse for a typical case); if they were smaller, we should see a much narrower distribution in the vertical direction. The effect of extinction is present but is much smaller: the yellow line shows the extinction trajectory for $A_{1}=0.1$ mag assuming a power-law NIR extinction $A_{\lambda}=A_{1} \lambda^{-\alpha}$ with $\alpha=2.0$. We note that this is about four times the expected
Table 3. Results for the FGK dwarf sample.

\begin{tabular}{lcc}
\hline \hline ZPs & $\left\langle A_{1}\right\rangle$ & $\sigma_{A_{1}}$ \\
\hline Cohen et al. (2003) & -0.055 & 0.257 \\
Holberg \& Bergeron (2006) & +0.203 & 0.243 \\
Maíz Apellániz (2007) & +0.101 & 0.252 \\
This work & +0.012 & 0.258 \\
This work $(\log Z=-0.5)$ & +0.049 & 0.250 \\
\hline
\end{tabular}

effect of $A_{1}=0.024 \mathrm{mag}$ for the sample, which for this extinction law corresponds to $E(H-K)=0.004 \mathrm{mag}^{4}$.

For each target we calculate $A_{1}$ by tracing back the extinction trajectory to the intrinsic color curve derived from the Brott \& Hauschildt (2005) models and we repeat it for the different ZPs in Table 2. Given that photometric uncertainties are the most important cause for dispersion in Fig. 2, many objects will have negative measured values of $A_{1}$. The results for the average $A_{1}$ are given in Table 3 . The ZPs in this work are those which are closer to the expected value of $0.024 \mathrm{mag}$. Cohen et al. (2003) yield an average negative value of $A_{1}$ (the blue curve is displaced upwards) while Holberg \& Bergeron (2006) and Maíz Apellániz (2007) yield average values of $A_{1}$ that are too large (their curves are displaced downwards). The expected value lies between the cases in this work with solar and subsolar metallicity, as expected for lines of sight near the Galactic poles (switching from solar to subsolar metallicity increases $A_{1}$ by $0.037 \mathrm{mag}$ ). Indeed, if most of the extinction originates close to the Galactic plane, the population at short distances should have a higher average metallicity than that at long distances. For our method, that translates into a measured $A_{1}$ that artificially decreases with distance (if one does not correct for metallicity) and we indeed see that effect in our data.

We conclude that our ZPs provide a better fit to the intrinsic NIR colors of FGK stars than previous works. We note, however, that differences between different sets of ZPs are relatively small: the small ellipse in Fig. 2 shows that the ZPs of Maíz Apellániz (2007) are within 1 sigma (in the color-color plane) and those of the other two works are not far away. Even though we claim our results are better than previous ones and should be used preferentially, there are no large fundamental differences between the four calibrations. The reasons why there is not a better possible calibration of the 2MASS at this stage are two: on the one hand, the amount of CALSPEC data is limited and, alas, with NICMOS long gone there will be no more high-quality HST spectroscopy in the whole $1.0-2.5 \mu \mathrm{m}$ range. On the other hand, 2MASS photometry saturates at 4-5 magnitudes and brighter stars in the survey have large uncertainties that make them useless for calibration purposes. With the overall interest of the astronomical community focussed on going deeper and deeper in magnitude, we are forgetting the brighter stars which are not only interesting by themselves but are also the best calibration targets given that their high fluxes yield large signal to noise ratios (S/Ns) in short exposure times for most programs. The problem with bright stars in the NIR is that most detector setups saturate them even at the shortest exposure times, and so this is a case where we need very small telescopes. If it were not for the differences in the access to technology between

4 Strictly speaking, the value of $E(H-K)$ depends on the SED, as it is a filter-integrated quantity, but the differences are minimal in the NIR (much smaller than $1 \mathrm{mmag}$ ) for the stars considered here (Maíz Apellániz 2013; Maíz Apellániz \& Barbá 2018). 
the optical and the NIR, an excellent amateur astronomy project would be an all-sky NIR photometric survey.

Acknowledgements. This work has made use of data from: (a) The ESA/NASA Hubble Space Telescope, obtained from the data archive at the Space Telescope Science Institute (STScI). STScI is operated by the Association of Universities for Research in Astronomy, Inc. under NASA contract NAS 5-26555. (b) The Two Micron All Sky Survey (2MASS), which is a joint project of the University of Massachusetts and the Infrared Processing and Analysis Center/California Institute of Technology, funded by the National Aeronautics and Space Administration (NASA) and the National Science Foundation (NSF). For both institutions the word "National" refers to the United States of America. (c) The European Space Agency (ESA) mission Gaia (https://www. cosmos.esa.int/gaia), processed by the Gaia Data Processing and Analysis Consortium (DPAC, https://www. cosmos.esa.int/web/gaia/dpac/ consortium). Funding for the DPAC has been provided by national institutions, in particular the institutions participating in the Gaia Multilateral Agreement We acknowledge support from the Spanish Government Ministerio de Ciencia, Innovación y Universidades through grant AYA2016-75 931-C2-2-P. M.P.G acknowledges support from the ESAC Trainee program.

\section{References}

Bohlin, R. C. 2007, in The Future of Photometric, Spectrophotometric and Polarimetric Standardization, ed. C. Sterken, ASP Conf. Series, 364, 315 Bohlin, R. C., \& Cohen, M. 2008, AJ, 136, 1171

Bohlin, R. C., Mészáros, S., Fleming, S. W., et al. 2017, AJ, 153, 234

Brott, I., \& Hauschildt, P. H. 2005, in The Three-Dimensional Universe with Gaia, eds. C. Turon, K. S. O’Flaherty, \& M. A. C. Perryman, ESA Special Publication, 576, 565

Cohen, M., Wheaton, W. A., \& Megeath, S. T. 2003, AJ, 126, 1090

Finlator, K., Ivezić, Ž., Fan, X., et al. 2000, AJ, 120, 2615

Gaia Collaboration (Brown, A. G. A., et al.) 2018, A\&A, 616, A1

Holberg, J. B., \& Bergeron, P. 2006, AJ, 132, 1221

Luri, X., Brown, A. G. A., \& Sarro, L. M., 2018, A\&A, 616, A9

Maíz Apellániz, J. 2001, AJ, 121, 2737

Maíz Apellániz, J. 2005a, PASP, 117, 615

Maíz Apellániz, J. 2005b, in The Three-Dimensional Universe with Gaia, eds C. Turon, K. S. O'Flaherty, \& M. A. C. Perryman, ESA Special Publication, 576, 179

Maíz Apellániz, J. 2006, AJ, 131, 1184

Maíz Apellániz, J. 2007, in The Future of Photometric, Spectrophotometric and Polarimetric Standardization, ed. C. Sterken, ASP Conf. Series, 364, 227

Maíz Apellániz, J. 2013, HSA7, 589

Maíz Apellániz, J. 2017, A\&A, 608, L8

Maíz Apellániz, J., \& Barbá, R. H. 2018, A\&A, 613, A9

Maíz Apellániz, J., Evans, C. J., Barbá, R. H., et al. 2014, A\&A, 564, A63

Schlafly, E. F., \& Finkbeiner, D. P. 2011, ApJ, 737, 103

Skrutskie, M. F., Cutri, R. M., Stiening, R., et al. 2006, AJ, 131, 1163

Weiler, M., Jordi, C., Fabricius, C., \& Carrasco, J. M. 2018, A\&A, 615, A24

\section{Appendix A: Zero points and conversions between magnitude systems}

In order to compare observed photometric magnitudes $\left(m_{\mathrm{phot}, \mathrm{p}}\right)$ in a series of filter passbands (denoted here by the $p$ index) with spectral energy distributions (SEDs, denoted here by the $s$ index) $f_{\lambda, \mathrm{s}}(\lambda)$ one has to define a magnitude system $r$ and compute synthetic magnitudes $\left(m_{\mathrm{r}, \mathrm{p}}\right)$ from the SEDs. For a photon-counting detector and a total-system dimensionless sensitivity function $P_{\mathrm{p}}(\lambda)$

$m_{\mathrm{r}, \mathrm{p}}\left[f_{\lambda, \mathrm{s}}(\lambda)\right]=-2.5 \log _{10}\left(\frac{\int P_{\mathrm{p}}(\lambda) f_{\lambda, \mathrm{s}}(\lambda) \lambda \mathrm{d} \lambda}{\int P_{\mathrm{p}}(\lambda) f_{\lambda, \mathrm{r}}(\lambda) \lambda \mathrm{d} \lambda}\right)+\mathrm{ZP}_{\mathrm{r}, \mathrm{p}}$.

A magnitude system $r$ is defined by a reference $\operatorname{SED} f_{\lambda, \mathrm{r}}(\lambda)$ and a series of (relative) zero points $\mathrm{ZP}_{\mathrm{r}, \mathrm{p}}$ for each filter. Most current magnitude systems use one of these reference SEDs:

- Vega (or VEGAMAG): a measured Vega SED $f_{\lambda, \text { Vega }}$.

- ST: $f_{\lambda, \mathrm{ST}}=3.63079 \cdot 10^{-9} \mathrm{erg} \mathrm{s}^{-1} \mathrm{~cm}^{-2} \AA^{-1}$ (constant).
- $\mathrm{AB}: f_{\mathrm{v}, \mathrm{AB}}=3.63079 \cdot 10^{-20} \mathrm{erg} \mathrm{s}^{-1} \mathrm{~cm}^{-2} \mathrm{~Hz}^{-1}$ (constant). In principle, one can adjust the $f_{\lambda, \mathrm{r}}(\lambda)$ in such a way that $\mathrm{ZP}, \mathrm{r}, \mathrm{p}$ $=0.0$ for all filters, which generates the default or strict system for that SED. In practice, one calibrates observed magnitudes a posteriori and this results in (unavoidable) zero points, no matter what reference SED one uses ${ }^{5}$. If the analysis is done correctly the most one can expect is to have ZPs close to zero. For example, SDSS magnitudes use an AB reference system and have ZPs measured in hundredths of a magnitude ${ }^{6}$.

The 2MASS magnitude system was defined using Vega as the reference SED and, indeed, the ZPs we measure in this work are close to zero, as expected. However, Vega-based magnitudes have two problems. The first one is that the published Vega SEDs differ from one another, so any published work must clearly state which one is being used and make it available. In this paper we do so in the introduction. Strictly speaking, the accuracy (i.e., proximity to the real one) of the Vega SED being used does not matter significantly, as a change in the denominator in Eq. (A.1) is simply offset by a change in $\mathrm{ZP}_{\mathrm{r}, \mathrm{p}}$. The second problem surrounds the question of why one should use a Vega SED and not use a simpler ST or AB reference SED instead. From the point of view of designing a magnitude system from scratch, this is a valid issue and is the reason why more modern systems such as SDSS have taken that step. From the point of view of calibrating a preexisting photometric system, following that strategy leads to ZPs that are not close to zero and are a possible source of confusion. For that reason, the ZPs in this paper are expressed using Vega as a reference SED. Nevertheless, a valid point is that the Vega SED used may not be available so it should be possible to work with ST or AB magnitudes even for older photometric systems. The solution to this problem is given by Maíz Apellániz (2007): To convert from a Vega system to an ST system one should use their Eq. (4) and to do the same to an AB system one should use their Eq. (5). The ZPs for 2MASS are the ones in Table 2 here and the ST and AB magnitudes of the Vega SED (which is the same in both papers, hence the advantage of using the same Vega SED throughout the years) for the three 2MASS filters are given in Table 4 of Maíz Apellániz (2007). We note, however, that the synthetic $\mathrm{AB}$ magnitudes calculated in this way are in the default $\mathrm{AB}$ system $(\mathrm{ZP}=0)$, so they do not correspond to the observed photometric magnitudes. An alternative method is given in the appendix of Maíz Apellániz \& Weiler (in prep.).
5 An alternative to this technique is used in some instances for HST photometry, where the ZPs are forced to be zero and the changes are introduced in the photometric reduction pipeline to change the observed photometric magnitudes instead of the synthetic ones. The problem with this approach is that it introduces a source of confusion, as the pipeline may deliver a given magnitude for an observation of a star today and a different one for the same observation tomorrow.

6 See https://www.sdss.org/dr12/algorithms/fluxcal/ 